\title{
Development of a Printed Coil for Wirelessly Charging a Tracking Elderly Patch
}

\author{
Bouchta Hajjine ${ }^{1,2}$, Christophe Escriba ${ }^{1,2}$, Samuel Charlot ${ }^{1}$, Anne Hemeryck ${ }^{1}$, \\ Julien Roux ${ }^{1,2}$, Sabeha Fettouma Zedek ${ }^{1}$, Jean-Yves Fourniols ${ }^{1,2}$ \\ ${ }^{1}$ CNRS, LAAS, Toulouse, France \\ ${ }^{2}$ Univ. de Toulouse, INSA, Toulouse, France \\ Email: bhajjine@laas.fr, cescriba@laas.fr, scharlot@laas.fr, ahemeryc@laas.fr, jroux@laas.fr, sfzedek@laas.fr, \\ fourniol@laas.fr
}

Received 14 January 2016; accepted 16 April 2016; published 19 April 2016

Copyright (C) 2016 by authors and Scientific Research Publishing Inc.

This work is licensed under the Creative Commons Attribution-NonCommercial International License (CC BY-NC).

http://creativecommons.org/licenses/by-nc/4.0/

(c) (i) (8) Open Access

\begin{abstract}
Monitoring systems for elderly require a compromise between reduced size and operational autonomy. The latter to get a system as independent as possible and to fit with our application needs for daily use. Our patch is developed as a surveillance system for old people; we chose to equip it with a wireless charging system for greater ease of use, imperceptible in the everyday life and waterproofing. This paper presents the development of a printed receiver coil to be integrated in a wireless charger to be used in an elderly tracking patch. The proposed design is validated using simulation that presents a good agreement with measurement results: Simulation (@150 KHz: L = $10.74 \mu \mathrm{H} ; \mathrm{R}=3 \Omega$ ) and Measurement (@150 KHz: $\mathrm{L}=10.8 \mu \mathrm{H} ; \mathrm{R}=3.16 \Omega$ ). The receiver coil is elaborated on a polyimide substrate in the cleanroom of our laboratory LAAS-CNRS (Laboratory for Analysis and Architecture of Systems-National Center for Scientific Research) and a PCB (Printed Circuit Board) charger prototype is fabricated to test its performances before the integration of the wireless charging property in the tracking patch. The proposed coil presents a good compromise between small size and efficiency. For a charging current of $7.5 \mathrm{~mA}$, this coil can ensure the recharging of the patch up to a distance of $4.8 \mathrm{~mm}$ between the $Q i$ transmitter and receiver which is more than enough for our application.
\end{abstract}

\section{Keywords}

Wireless Charger, Receiving Coil, Tracking Patch, Cleanroom Process

\section{Introduction}

Wireless power transfer (WPT) was first demonstrated in the last decade of the $19^{\text {th }}$ century by Nikola Tesla

How to cite this paper: Hajjine, B., Escriba, C., Charlot, S., Hemeryck, A., Roux, J., Zedek, S.F. and Fourniols, J.-Y. (2016) Development of a Printed Coil for Wirelessly Charging a Tracking Elderly Patch. Wireless Engineering and Technology, 7, 83-95. http://dx.doi.org/10.4236/wet.2016.72009 
whose initial experiment was based on lighting wirelessly a group of lamps from over 25 miles away. However it is only recently that this concept has become the interest center in several fields due to the important added value provided by this technology in various highly applicative sectors such as the electric vehicles [1] [2], the implanted medical devices that can be charged wirelessly to avoid a surgical operation to replace a battery [3]-[4], or also for the portable electronic devices like mobile phones, an universal charging pad can resolve the problem of huge amount of incompatible and bulky chargers [5]-[9]. The WPT sector has witnessed the creation of some consortia that have developed various standards to define rules and requirements of systems for the wireless chargers [10] like the Wireless Power Consortium (WPC) [11], the Power Matters Alliance (PMA) [12], and the Alliance For Wireless Power (A4WP) [13].

In the current paper, we introduce the development of a printed charging coil to be integrated in a tracking patch for elderly suffering from Alzheimer disease [14]. Qi compatible charging systems are already present in the market. However, the available coils are bulky and not suitable for applications with specific dimensions constraints. Using a rod coil as the Qi charger presented in [15] can be opted for some systems but for the miniaturization of our patch, a very tiny coil must be used. This condition cannot be met with copper wires coils and this type is only presented in this article for performances comparison. To be suitable with batch production, we have opted to develop a 2-layers printed coil. There are many types of printed coils in the literature [16]-[19]. Some examples use a frequency below $13.56 \mathrm{MHz}$ for medical implantable devices. For our system, the frequency is fixed by the Qi protocol $(110$ - $205 \mathrm{KHz})$ and the proposed coil is characterized by a very small thickness due to the use of polyimide substrate $(50 \mu \mathrm{m})$ compared to FR4 used in [17] for example. Besides, it presents a good compromise between small dimensions and performances. Printed coils with polyimide substrate can be carried out using inkjet printing [20], laminated copper [21] and screen printing [22]. For this paper, a photoresist polyimide is used to avoid the vias drilling and get the different openings directly by photolithography to simplify the cleanroom process.

The second section of this paper gives more details on the wireless power standard defined by the WPC, the design of the coil is described in the third part, the fourth one describes the cleanroom process and finally we list measurement results and conclusion.

\section{QI Wireless Power Standard}

With the aim to ensure a wide use of the wireless power systems, the Wireless Power Consortium has developed an international standard named “Qi” to provide interoperability between different devices and chargers [23] [24]. This protocol defines an ensemble of guidelines and references for inductive power systems. By this way, one wireless charger can be easily used for many products incoming from different manufacturers. The WPC was established in December 2008 and contains various members from different industrial sectors including mobile phones, consumer electronics, batteries...

An inductive power system is composed of two parts: a transmitter (pad) and a receiver (mobile device) that are equipped with two coils to transfer power, when they are close from each other.

Figure 1 is a schematic picture of a contactless charging system. Charging energy is transferred across an inductive coupler to charge the battery. Coils of transmitter and receiver are electrically isolated from each other. Energy transfer is ensured by electromagnetic coupling known as the induced current name.

The coupling coefficient " $k$ " between these coils is a key parameter that defines the efficiency of the charging system. It must be the highest to ensure a reliable link between the pad and the receiver and thus a powerful transfer. The Qi standard defines three techniques to achieve an optimal alignment of the transmitting and the

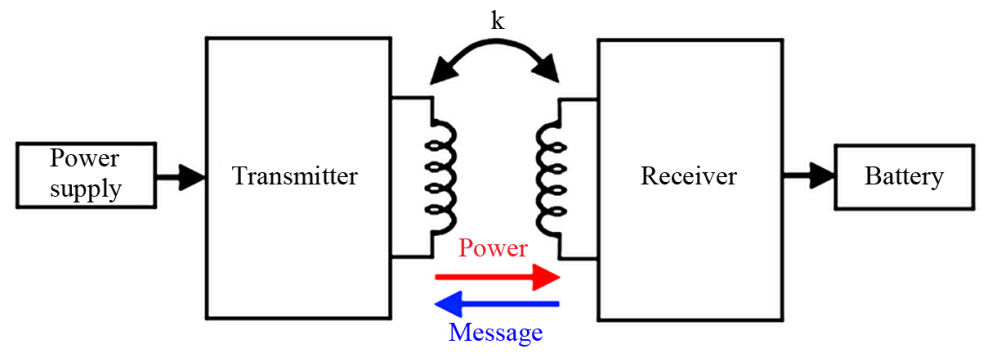

Figure 1. Principle of inductive wireless power transfer. 
receiving coils in order to enhance the yield of the wireless charging: guided positioning, free positioning with moving coil, and free positioning with coils matrix. In the guided one, the user is helped to achieve a good alignment with some indications to obtain an optimal coupling. The free positioning can be ensured by using a moving coil in the transmitter system which is able to locate the receiver coil and to move to be aligned with it. When using a matrix of coils, the transmitter activates the three nearest coils to supply power to the receiver.

For low power chargers (5W), Qi uses a frequency between 110 and $205 \mathrm{kHz}$ and provides also a one-direction communication from the receiver to the transmitter to control the different charging status. This link permits to exchange different information like the presence of a Qi compatible receiver, the full charging of the battery... This communication is based on a load modulation technique: the receiver changes its impedance by switching a resistor or capacitor. As a result the current or the voltage in the transmitting coil is modulated and different messages are transmitted in the form of differential bi-phase encoded bits with a period of $0.5 \mathrm{~ms}$.

\section{Design of the Printed Receiving Coil}

\subsection{Inductance of a Printed Rectangular Coil}

Based on Grover's equations [25], Greenhouse presented an algorithm for calculating the inductance of a printed rectangular coil [26], in which a spiral coil can be seen as an ensemble of $\mathrm{N}$ straight segments connected in series.

Figure 2 presents an example of a printed coil composed of 8 segments whose total inductance can be calculated as the sum of the self-inductance of each segment and the different negative and positive mutual inductances between them.

This method was implemented in MATLAB software [27] and compared to the simulation results obtained with ADS (Advanced Design system) RF software [28]. Figure 3 shows a graphic interface that permits to define the different parameters of a spiral printed coil and then compute its total inductance.

Table 1 provides results of different coils with the comparison of the computed inductances using Greenhouse's algorithm and the simulated values given by ADS. Both methods show very similar results. Here, we validate the Greenhouse's approach to be applied to our study.

\subsection{Design of the Wireless Charging Coil}

The receiving coil was designed on a polyimide substrate with a thickness of $50 \mu \mathrm{m}$. This flexible substrate is

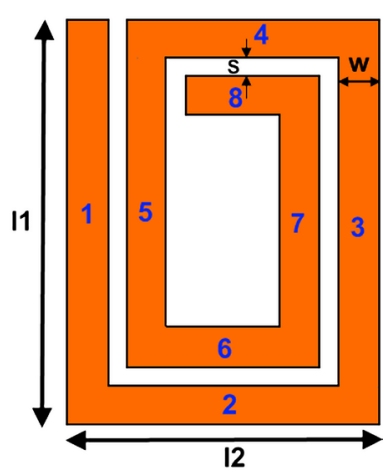

Figure 2. Rectangular spiral coil composed of 8 conductor segments.

Table 1. Comparison of the results given by Greenhouse's algorithm and ADS.

\begin{tabular}{ccccccccc}
\hline $\begin{array}{c}\text { Number } \\
\text { of turns }\end{array}$ & $\begin{array}{c}\text { Number of } \\
\text { segments }\end{array}$ & $\begin{array}{c}\mathrm{l} 1 \\
(\mathrm{~mm})\end{array}$ & $\begin{array}{c}\mathrm{l} 2 \\
(\mathrm{~mm})\end{array}$ & $\begin{array}{c}\mathrm{w} \\
(\mu \mathrm{m})\end{array}$ & $\begin{array}{c}\mathrm{t} \\
(\mu \mathrm{m})\end{array}$ & $\begin{array}{c}\mathrm{s} \\
(\mu \mathrm{m})\end{array}$ & $\begin{array}{c}\mathrm{L} \\
(\mu \mathrm{H})\end{array}$ & $\begin{array}{c}\mathrm{L} \_\mathrm{ADS} \\
(\mu \mathrm{H})\end{array}$ \\
\hline $\mathbf{3}$ & 12 & 25 & 10 & 400 & 35 & 150 & 0.328 & 0.325 \\
$\mathbf{8}$ & 32 & 32 & 18 & 300 & 70 & 100 & 2.89 & 2.88 \\
15 & 60 & 40 & 25 & 200 & 40 & 50 & 14.11 & 14.09 \\
25 & 100 & 60 & 40 & 500 & 100 & 200 & 22.8 & 22.63 \\
\hline
\end{tabular}




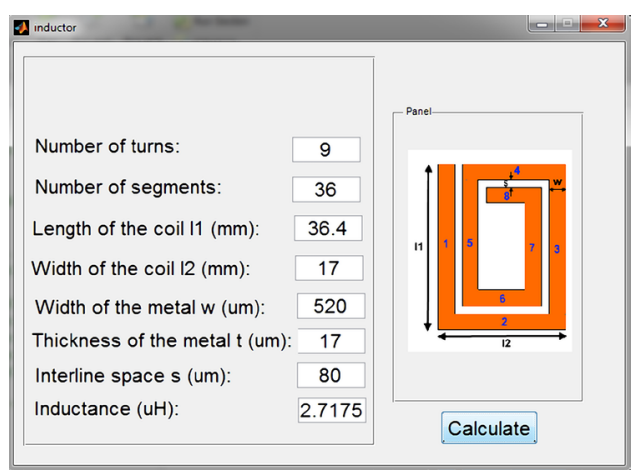

Figure 3. MATLAB graphic interface.

characterized by a good combination of electrical, chemical, and mechanical properties. As presented in Figure 4 our printed coil is composed of two copper layers and has a total dimension of $42.3 \times 17 \mathrm{~mm}^{2}$. This area is imposed by the integration requirements in the tracking patch. The coil tracks are made of copper with a thickness $\mathrm{t}=17 \mu \mathrm{m}$, a width $\mathrm{w}=520 \mu \mathrm{m}$, and an interline spacing $\mathrm{s}=80 \mu \mathrm{m}$.

Our interest frequencies range is located between 110 and $205 \mathrm{KHz}$. A first approximate value of the inductance of the receiver coil can be given by using the Greenhouse's algorithm. The first layer is composed of 9 turns and 36 straight conductor segments that provide an inductance of $2.72 \mu \mathrm{H}$ (Figure 3). For the total inductance of the two layers and as the thickness of the polyimide substrate is less than the interline spacing [29] [30], it is about 4 times the inductance of the first layer: $\mathrm{L}=10.88 \mu \mathrm{H}$ compared to $10.74 \mu \mathrm{H}$ obtained with ADS (Figure 5).

The proposed coil has an inductance of $10.74 \mu \mathrm{H}$ and a resistance of about $3 \Omega$, right in the targeted frequencies range. From Figure 5, we can see that the resonance frequency (5.3 MHz) is located far of this range, indicating that this coil can be used for the charging system.

\section{Cleanroom Process Description}

The proposed coil is composed of two copper layers connected by vias. The other layers are made with polyimide used as a negative photoresist HD-4110 [31] that allows to design different openings (vias and connection pads) by photolithography. This photoresist can be deposited by spin coating and permits to get a cover layer with a thickness of $9 \mu \mathrm{m}$ and a $56 \mu \mathrm{m}$ polyimide substrate. A sacrificial layer of a non-photosensitive polyimide PI2611 [32] was added between the coil and the wafer to facilitate their separation at the end of the process. The conductor tracks were carried out by electroplating based on a seed layer of $\mathrm{Ti} / \mathrm{Cu}(100 / 200 \mathrm{~nm})$ deposited by evaporation and then removed by chemical etching. A BPN photoresist was used to delimit the areas for the electroplating process and form the conductive lines of the coil. This method provides directly the desired shape for the copper layer and avoids difficulties associated with copper etching. Steps of the process flow to elaborate the first copper layer are schematized on Figure 6.

The first copper tracks with a thickness of $18 \mu \mathrm{m}$ obtained using the process described in Figure 6 are shown in Figure 7.

After the setting up of the first conductive tracks, a polyimide layer was deposited and insolated to form the contact vias (Figure 8). The proposed receiver coil contains four large vias to ensure the connection between the pads of the two copper layers and two small ones to link the $\mathrm{Cu}$ lines.

Figure 9 summarizes the process steps to form the second copper layer which is ended up using the same method as described for the first conductive tracks. At the end another polyimide cover layer was added to protect the Cu paths.

The proposed receiving coil is presented in Figure 10. The different pads, vias, and copper tracks were checked to avoid any discontinuity or short circuit that could affect the coil performances.

\section{Test of the Proposed Receiving Coil}

\subsection{Inductance Measurement}

Two prototypes were fabricated and characterized using an impedance analyzer Agilent 4294A (Figure 11) to 

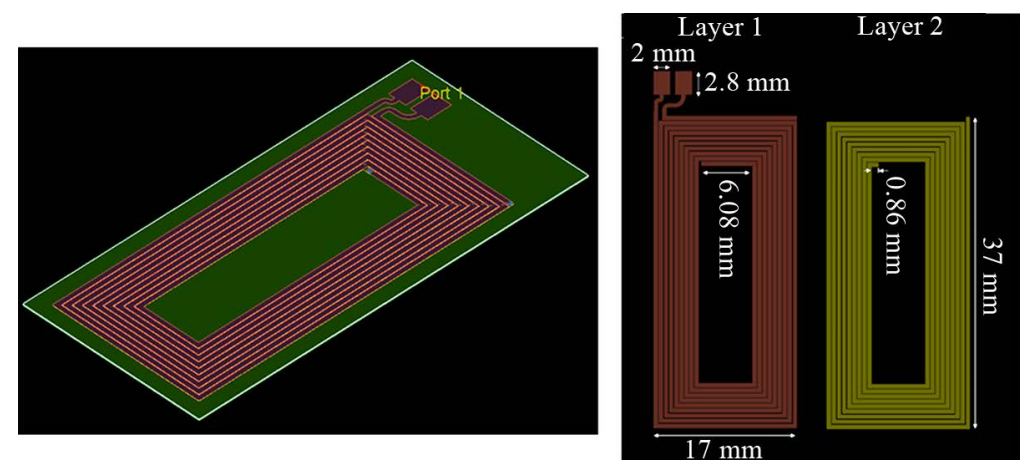

Figure 4. Design of the proposed receiving coil with ADS.

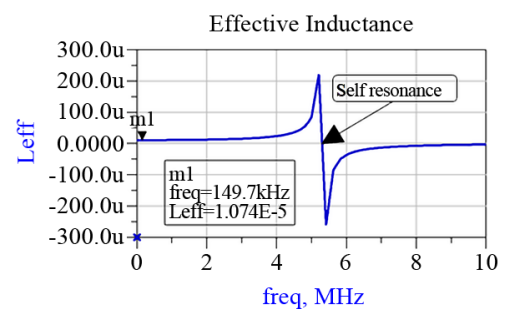

(a)

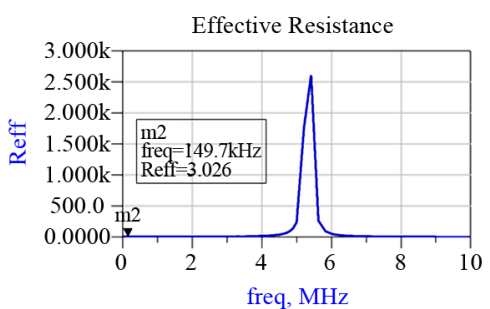

(b)

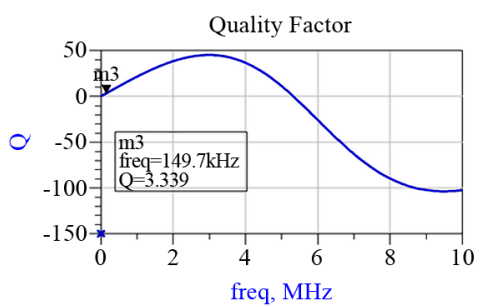

(c)

Figure 5. Simulation results obtained with ADS: (a) Inductance $(\mu \mathrm{H})$, (b) Resistance $(\Omega)$, and (c) Quality factor.

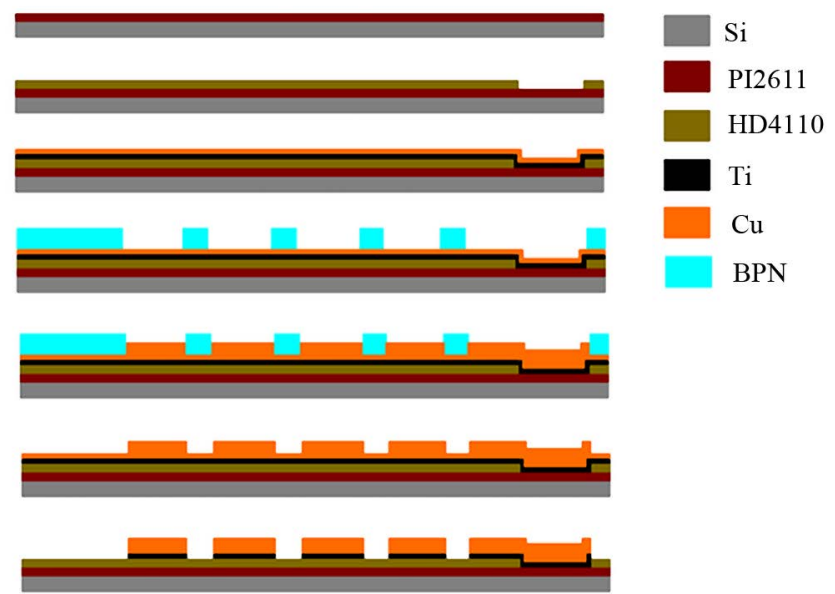

Figure 6. Process steps of the first conductive layer.
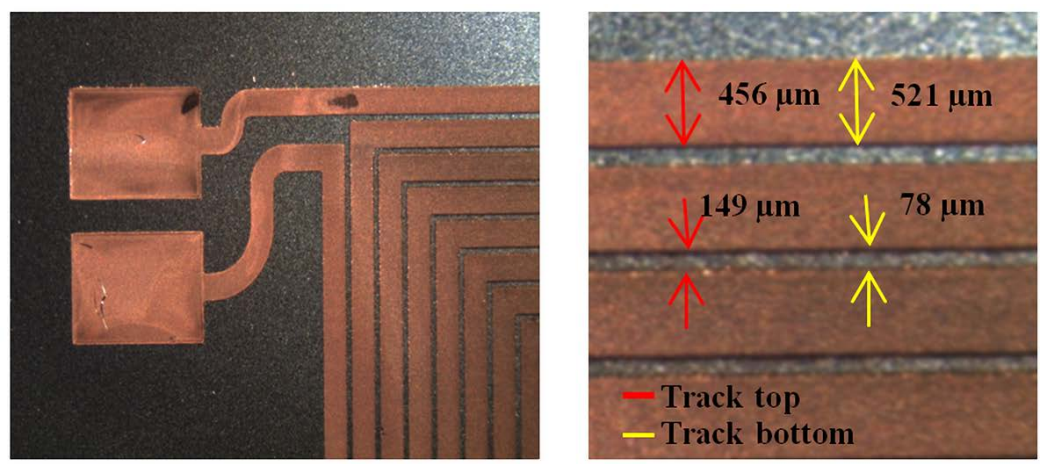

Figure 7. Profiles of the first Cu layer tracks. 


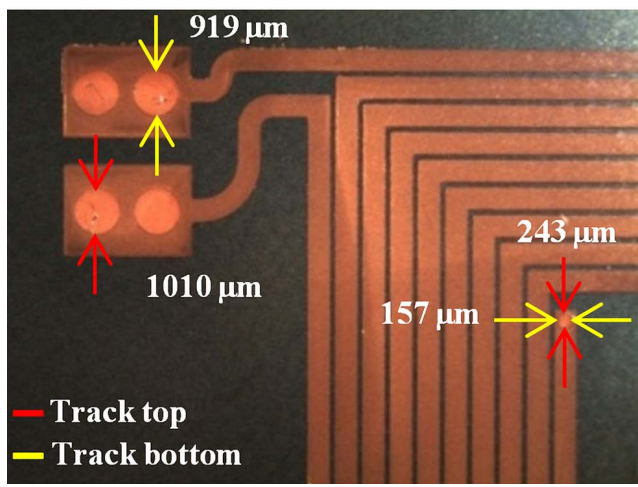

Figure 8. Profiles of the connection vias.
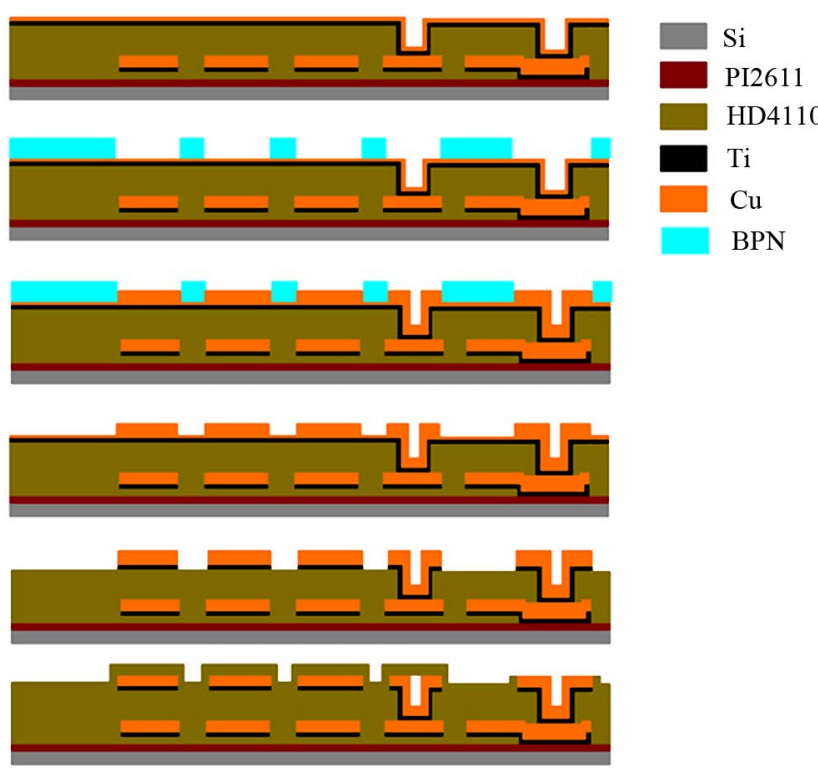

Figure 9. Process steps of the second copper layer.
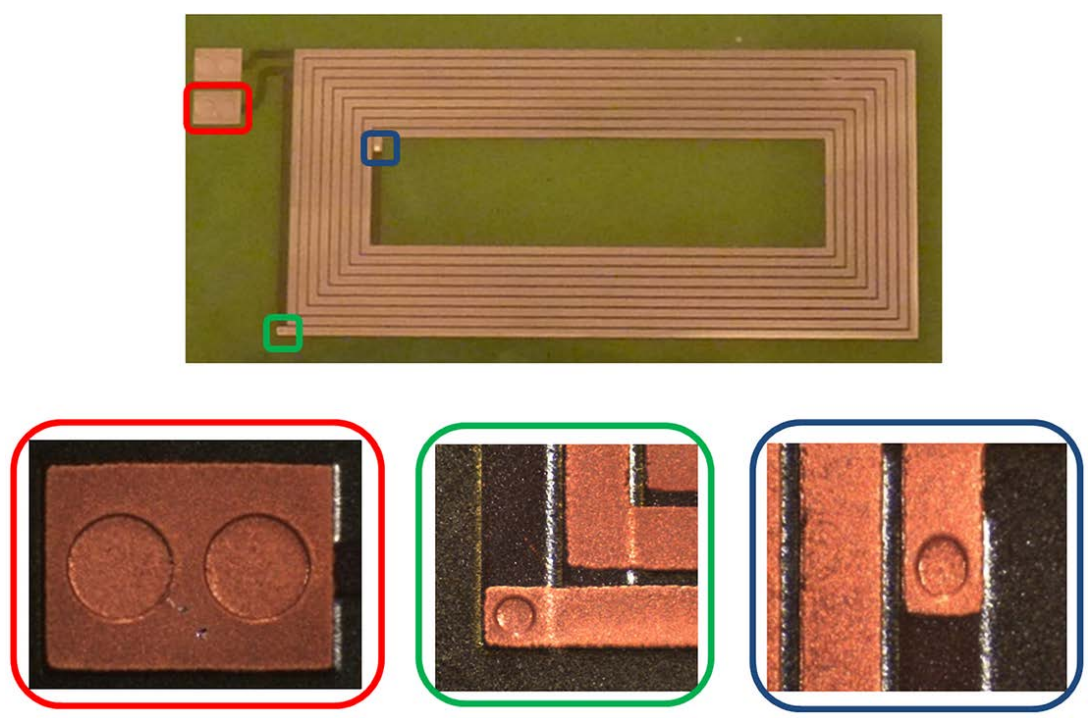

Figure 10. Proposed coil and different connection vias. 


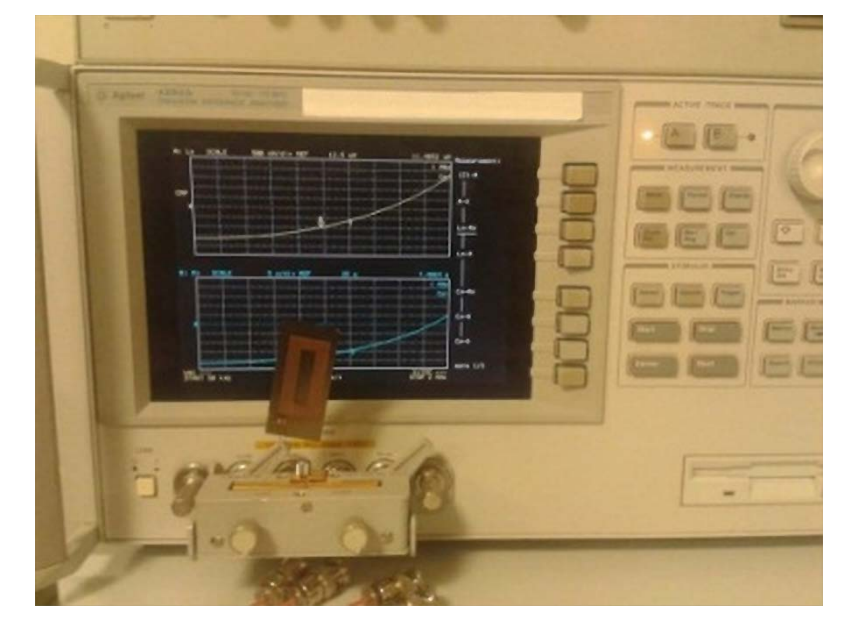

Figure 11. Characterization of the printed coil based on Agilent 4294A.

get the inductance, resistance, and quality factor values.

The measurements results are plotted in Figure 12. For the range of $110-205 \mathrm{KHz}$, the proposed printed coil provides an inductance of $10.8 \mu \mathrm{H}$ and a resistance of $3.16 \Omega$ that fit well with the simulated values. We can see that the resonance frequency $(3.8 \mathrm{MHz}$ ) is shifted compared to the simulated curves (5.3 MHz). Differences between simulated and measured peak and minimum values are also noticed (Table 2). However, they still far of the working frequency domain used by the Qi protocol and there is no influence on the coil charging performances.

Because of a thin-thick copper tracks integration $(18 \mu \mathrm{m})$, the quality factor is small in comparison with the marketed coils. Choosing a larger thickness value will reduce the total resistance and improve the quality factor. The next step is to test the proposed coil with a Qi wireless charger.

\subsection{Test of the Wireless Charging System}

In order to be compatible with the Qi standard a receiver that already exists on the market was used, it is the BQ51050B from Texas Instrument [33]. This component regroups a wireless power receiver and Li-Ion/Li-Pol battery charger.

Figure 13 describes the test of the wireless power transfer using a commercial charging pad compatible with the Qi protocol. The charging current is measured using an oscilloscope DPO4034 and a current probe TCP0030A from TEKTRONIX. An example of this current with a value of $22 \mathrm{~mA}$ is presented in Figure 14.

After the validation of the wireless charger, it was integrated in our tracking patch. The integrated system with the packaging to ensure the waterproof characteristics is presented on Figured 15.

The patch is equipped with a tiny battery characterized by a capacity of $30 \mathrm{mAh}$. The charging current was adjusted to $7.5 \mathrm{~mA}$, providing a charging time of $4-5$ hours.

A ferrite layer was also added between the receiving coil and the battery to avoid perturbations in the magnetic field lines and ensure an optimum energy transfer as depicted in Figure 16.

Another test performed on the efficiency of the charging system using the tracking patch and the stacking of the different layers (Figure 16) was carried out. This test consists in measuring the ratio between the DC power at the receiver output and the DC power consumed by the charging pad. Another prototype of the printed coil was added with a $\mathrm{Cu}$ layer thickness of $35 \mu \mathrm{m}$ to check the influence of the quality factor on the charging efficiency and two other types of coils were selected for the test and compared with our coils (Figure 17): The first one is a marketed coil referred as WR483250-15M2-G from TDK [34] and the second one is handmade using two copper wires in parallel.

The comparison of the performances of the different coils used in the efficiency test is shown in Figure 18. Except the marketed coil, all the other coils were tested with the patch layers stacking presented in Figure 16 to take into account the influence of all parameters during the integration.

The variation of the efficiency as a function of the charging current is presented in Figure 19. For this test, the distance between the charging pad and the receiving coil was fixed to $1.6 \mathrm{~mm}$. 


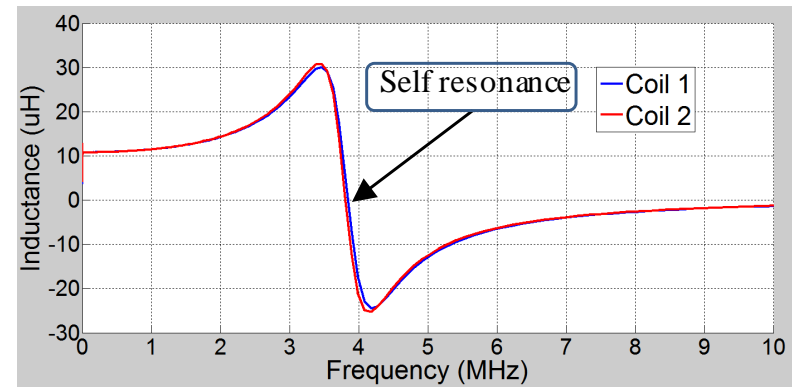

(a) Inductance $(\mu \mathrm{H})$

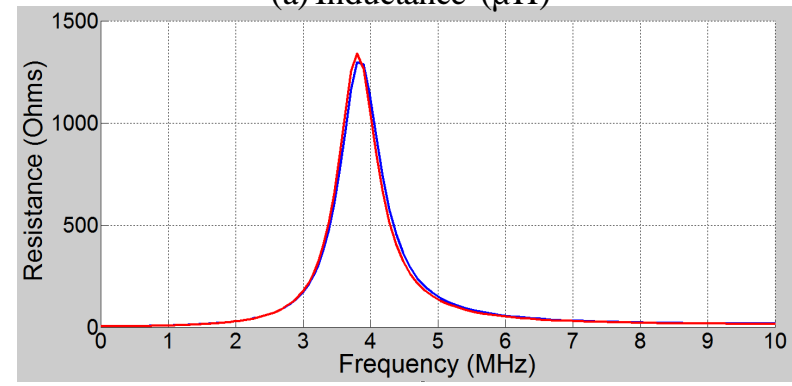

(b) Resistance $(\Omega)$

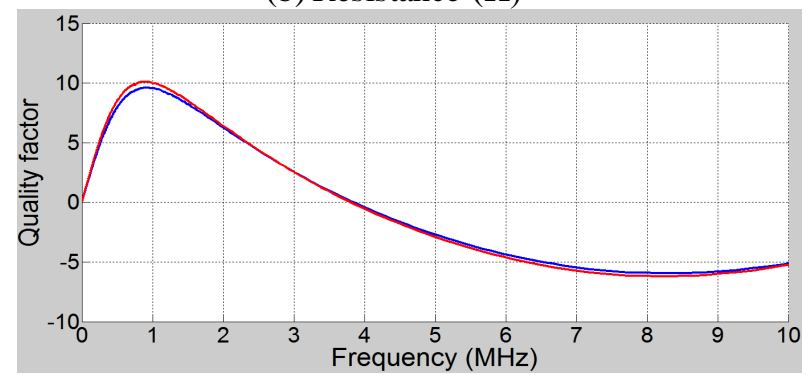

(c) Quality factor

Figure 12. Characterization of the printed coil based on Agilent 4294A.

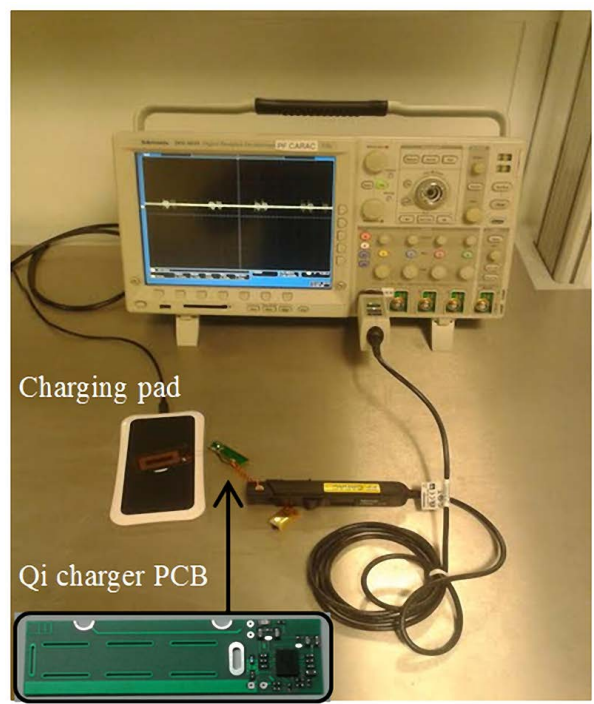

Figure 13. Test of the receiving coil with a Qi compatible charger. 


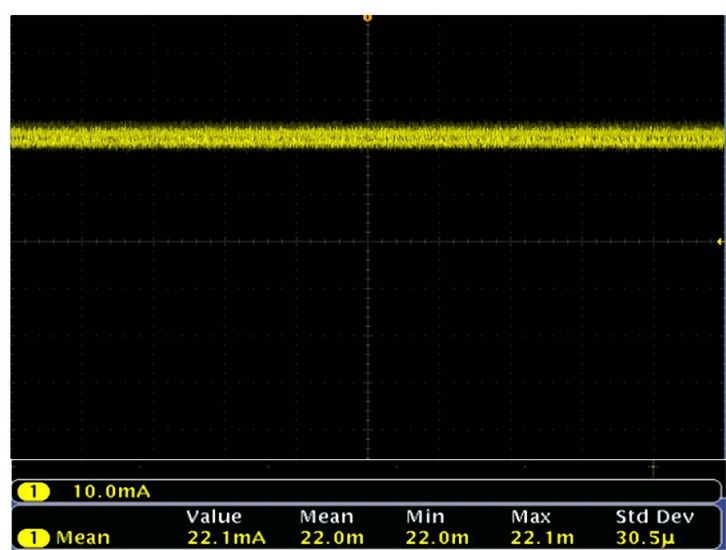

Figure 14. Charging current.
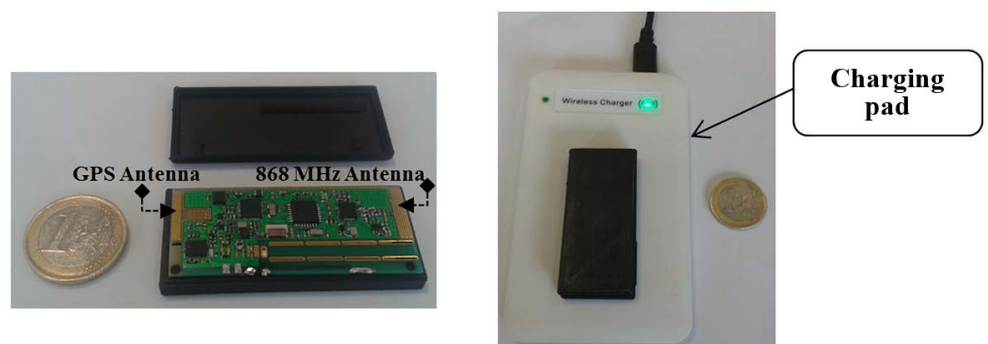

Figure 15. Tracking patch with waterproof packaging and integrated wireless charger.

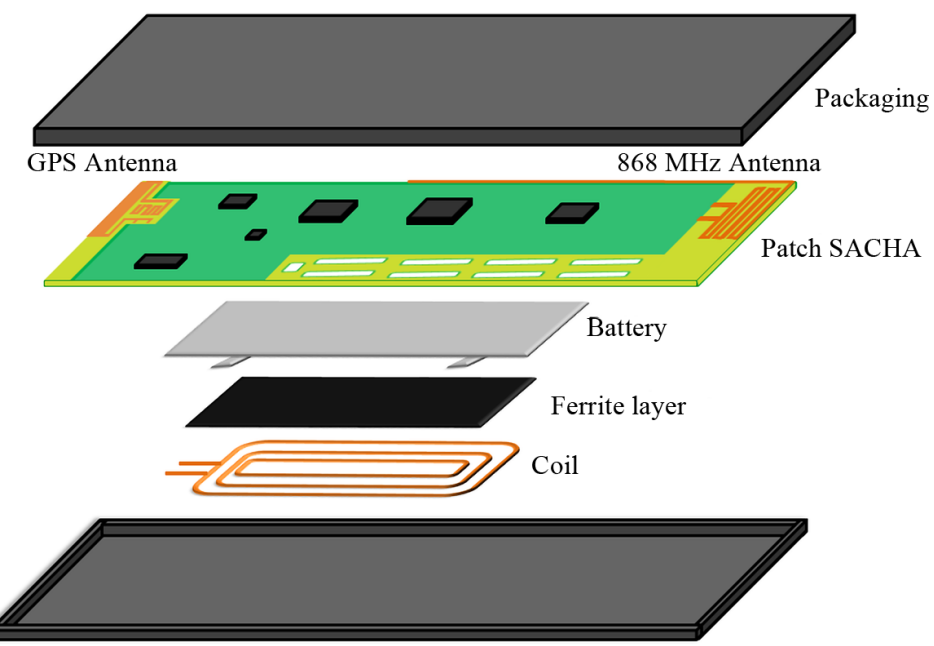

Figure 16. Stacking of the different tracking patch layers.

Table 2. Comparison of the different simulated and measured peak and minimum values of the printed coil performances.

\begin{tabular}{ccccccc}
\hline & \multicolumn{3}{c}{ Peak value } & \multicolumn{3}{c}{ Minimum value } \\
\cline { 2 - 7 } & Simulation & Coil 1 & Coil 2 & Simulation & Coil 1 & Coil 2 \\
\hline Inductance $(\mu \mathrm{H})$ & 221 & 30.1 & 30.8 & -261 & -24.5 & -25.27 \\
Resistance $(\Omega)$ & 2614 & 1296 & 1340 & 3.013 & 3.11 & 3.07 \\
Quality factor & 45.19 & 9.59 & 10.13 & -103.81 & -5.91 & -6.18 \\
\hline
\end{tabular}




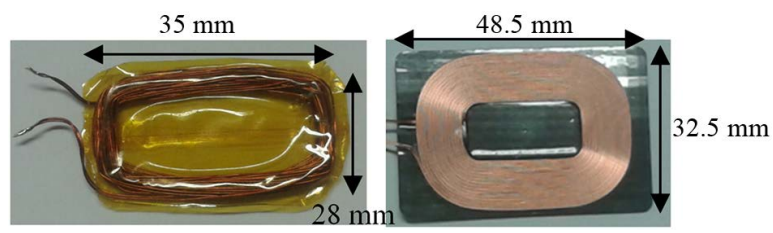

Figure 17. Handmade (left) and commercialized (right) coils.

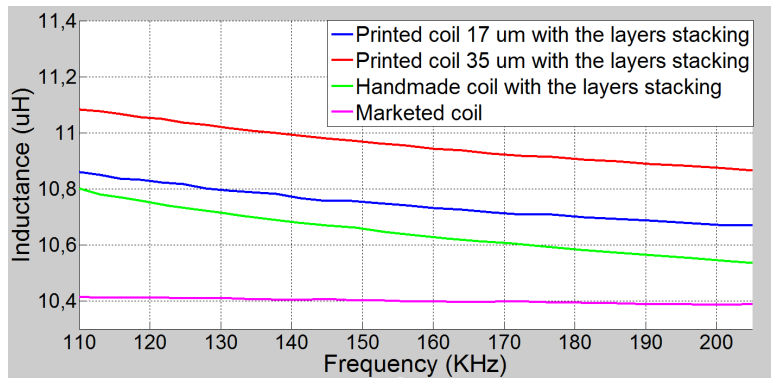

(a) Inductance $(\mu \mathrm{H})$

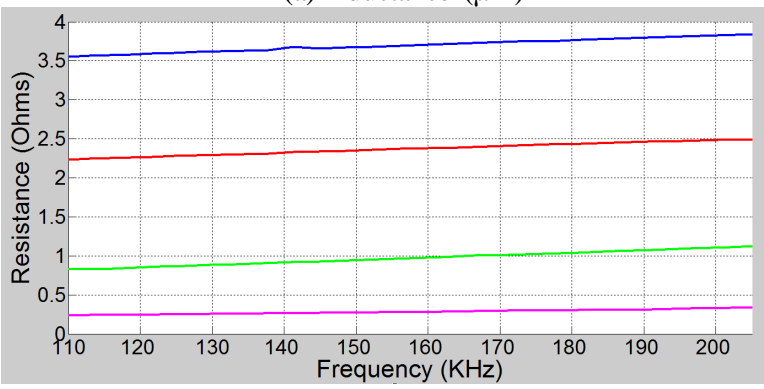

(b) Resistance $(\Omega)$

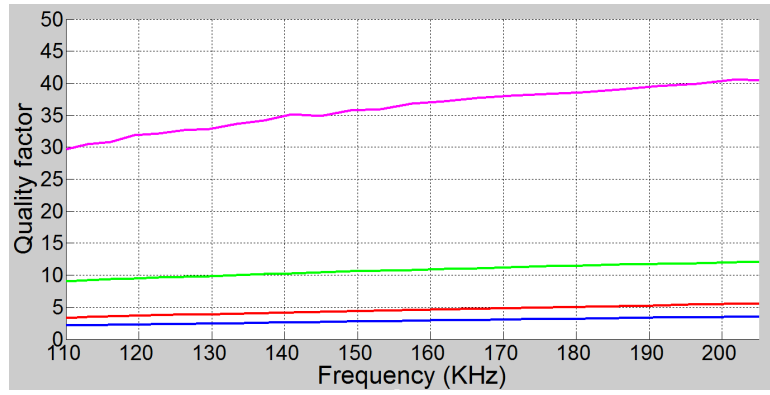

(c) Quality factor

Figure 18. Comparison of the different coil performances in the charging frequency range.

The efficiency of the wireless charger increases with the load current. Despite its small quality factor (Figure 18: many times smaller than that of the marketed coil), our printed coil achieves a maximal efficiency of $26 \%$ which represents $45 \%$ of that of the marketed one. In addition, this performance can be more improved by using thicker copper layers to reduce the resistance and improve the quality factor.

The plots of the efficiency according to the distance between the transmitting and the receiving coils are given in Figure 20. Two charging currents were chosen 7.5 and $300 \mathrm{~mA}$ and the distance was increased with a step of $1.6 \mathrm{~mm}$ from 0 to the maximal distance beyond which the system cannot ensure the recharging of the battery.

From Figure 20, we can notice that the efficiency of the wireless charging system does not vary a lot with the distance.

The developed and integrated coil does not exhibit currently the best efficiency, but it is characterized by a good compromise between small size and performances in comparison with the marketed coil. In the case of a 


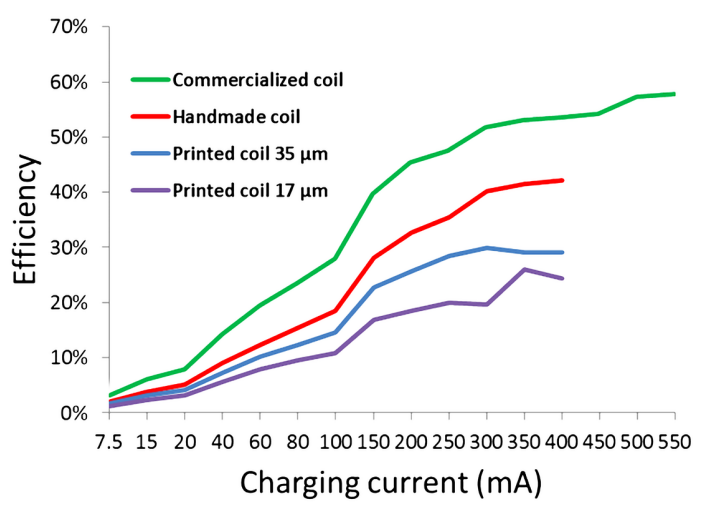

Figure 19. Efficiency of the wireless charger as a function of the load current.

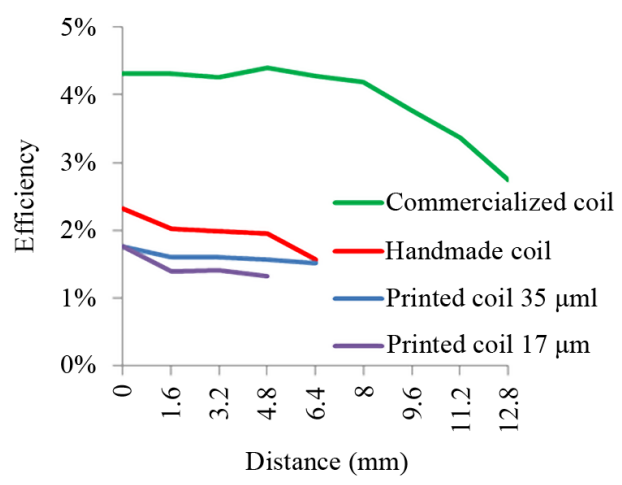

(a)

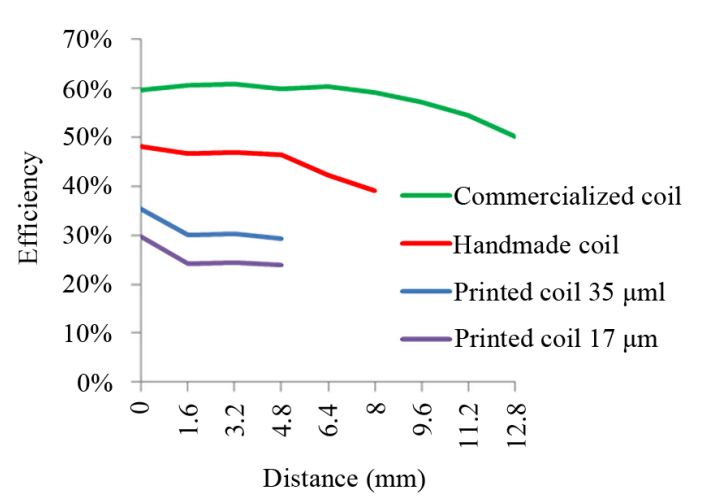

(b)

Figure 20. Efficiency of the wireless charger as a function of the distancebetween the transmitting and the receiving coils, charging currents: (a) $7.5 \mathrm{~mA}$ and (b) $300 \mathrm{~mA}$.

charging current of $7.5 \mathrm{~mA}$, this coil can ensure the recharging of the tracking patch up to a distance of $4.8 \mathrm{~mm}$ between the transmitter and receiver coils which is more than enough for our application. In addition our printed coil presents a high degree of integration compared to the bulky commercialized coils that cannot be used in our small system. This property permits to ensure invisibility, high miniaturization and furthermore a waterproof feature of the tracking patch when an adequate packaging is used.

\section{Conclusions}

Coils are essential components in an inductive charging system to ensure an efficient power transfer. Most marketed coils are made of copper wires and are bulky, so that they cannot be directly integrated in small systems like in a tracking patch aiming to be invisible in the everyday life as worn daily by elderly.

To overcome the integration constraint and enhance the size and weight requirements, we designed a printed and rectangular coil in the cleanroom of LAAS-CNRS, which has been integrated then in the receiver part of our tracking patch, as a Qi wireless charging system. The proposed coil is elaborated on a polyimide substrate. The developed coil is endowed with small dimensions compared to other charging coils available on the market with a good compromise between small size and charging efficiency that meets very well our application requirements. The chosen cleanroom process is characterized by its simplicity thanking to the use of a polyimide photoresist that enables us to get the needed pads and vias directly by photolithography and electroplating avoiding chemical etching problems. The measurement results meet the simulation values and the charging system is tested and validated with different Qi charging pads.

\section{Acknowledgements}

This work is part of SACHA project funded by French government and "Région Midi Pyrénées” in France. The 
partners of the project are Sigfox, Axible, Telecom Design companies, esanté and CHIVA hospital.

\section{References}

[1] Chun, Q., Chau, K.T., Liu, C.H. and Chan, C.C. (2013) Overview of Wireless Power Transfer for Electric Vehicle Charging. World Electric Vehicle Symposium and Exhibition (EVS27), Barcelona, 17-20 November 2013, 1-9.

[2] Siqi, L. and Mi, C.C. (2015) Wireless Power Transfer for Electric Vehicle Applications. IEEE Journal of Emerging and Selected Topics in Power Electronics, 3, 4-17. http://dx.doi.org/10.1109/JESTPE.2014.2319453

[3] Jolani, F., Mehta, J., Yu, Y. and Chen, Z. (2013) Design of Wireless Power Transfer Systems Using Magnetic Resonance Coupling for Implantable Medical Devices. Progress in Electromagnetics Research Letters, 40, 141-151. http://dx.doi.org/10.2528/PIERL13020509

[4] Majerus, S., Garverick, S.L. and Damaser, M.S. (2014) Wireless Battery Charge Management for Implantable Pressure Sensor. IEEE Dallas Circuits and Systems Conference (DCAS), Richardson, 12-13 October 2014, 1-5. http://dx.doi.org/10.1109/DCAS.2014.6965336

[5] Boscaino, V., Pellitteri, F., Capponi, G. and Rosa, R.L. (2012) A Wireless Battery Charger Architecture for Consumer Electronics. IEEE International Conference on Consumer Electronics-Berlin (ICCE-Berlin), Berlin, 3-5 September 2012, 84-88. http://dx.doi.org/10.1109/icce-berlin.2012.6336483

[6] Sebastian, E., Biji, B., Pranav, V., Rahul, A.P. and Nair, V.N. (2014) Inductive Charging Pad. International Journal of Advanced Research in Electrical, Electronics and Instrumentation Engineering, 3, 9549-9556.

[7] Olvitz, L., Vinko, D. and Svedek, T. (2012) Wireless Power Transfer for Mobile Phone Charging Device. Proceedings of the 35th International Convention on MIPRO, Opatija, 21-25 May 2012, 141-145.

[8] Boscaino, V., Pellitteri, F., Rosa, L. and Capponi, G. (2013) Wireless Battery Chargers for Portable Applications: Design and Test of a High-Efficiency Power Receiver. IET Power Electronics, 6, 20-29. http://dx.doi.org/10.1049/iet-pel.2012.0272

[9] Waffenschmidt, E. (2011) Wireless Power for Mobile Devices. 33rd IEEE International Telecommunications Energy Conference (INTELEC), Amsterdam, 9-13 October 2011, 1-9. http://dx.doi.org/10.1109/intlec.2011.6099840

[10] Pathare, N. (2013) A Global Wireless Power Standard Will Open the Market, Encourage Consumers to Live without Power Cords. http://www.ti.com/lit/wp/slyy036/slyy036.pdf

[11] Wireless Power Consortium. http://www.wirelesspowerconsortium.com/

[12] Power Matters Alliance. http://www.powermatters.org/

[13] Alliance for Wireless Power. http://www.rezence.com/

[14] Hajjine, B., Escriba, C., Campo, E., Zedek, S., Acco, P., Soto Romero, G., Hemeryck, A. and Fourniols, J.Y. (2015) Development of an Electronic Patch for Falls Detection and Elderly Tracking. Proceedings of the International Conference on Biomedical and Health Informatics (ICBHI), Haikou, 9-10 October 2015. (in press)

[15] Solar, H., Alonso, M., Bustamante, P. and Giers, C. (2015) Design of a Wireless Power Transfer Receiver with an Ad-Hoc Coil for the Qi Standard. Conference on Design of Circuits and Integrated Systems (DCIS), Estoril, 25-27 November 2015, 1-5. http://dx.doi.org/10.1109/dcis.2015.7388586

[16] Duan, Z., Guo, Y.-X. and Kwong, D.-L. (2012) Rectangular Coils Optimization for Wireless Power Transmission. Radio Science, 47, RS3012. http://dx.doi.org/10.1029/2011rs004970

[17] Seo, D.-W., Lee, J.-H. and Lee, H. (2014) Integration of Resonant Coil and Antenna for Wireless Power Transfer and Data Telemetry. International Symposium on Antennas and Propagation (ISAP), Kaohsiung, 2-5 December 2014, 617-618. http://dx.doi.org/10.1109/isanp.2014.7026802

[18] Xue, R.-F., Cheng, K.-W. and Je, M. (2013) High-Efficiency Wireless Power Transfer for Biomedical Implants by Optimal Resonant Load Transformation. IEEE Transactions on Circuits and Systems I: Regular Papers, 60, 867-874. http://dx.doi.org/10.1109/TCSI.2012.2209297

[19] Jow, U.-M. and Ghovanloo, M. (2007) Design and Optimization of Printed Spiral Coils for Efficient Transcutaneous Inductive Power Transmission. IEEE Transactions on Biomedical Circuits and Systems, 1, 193-202. http://dx.doi.org/10.1109/TBCAS.2007.913130

[20] Mager, D., Peter, A., Del Tin, L., Fischer, E., Smith, P.J., Hennig, J. and Korvink, J.G. (2010) An MRI Receiver Coil Produced by Inkjet Printing Directly on to a Flexible Substrate. IEEE Transactions on Medical Imaging, 29, 482-487. http://dx.doi.org/10.1109/TMI.2009.2036996

[21] Waffenschmidt, E., Ackermann, B. and Wille, M. (2005) Integrated Ultra Thin Flexible Inductors for Low Power Converters. IEEE 36th Power Electronics Specialists Conference, PESC '05, Recife, 16 June 2005, 1528-1534. http://dx.doi.org/10.1109/pesc.2005.1581833 
[22] Yamashita, T., Zhang, Y., Itoh, T. and Maeda, R. (2014) Flexible Current Clamp Sensor Using Screen-Printed Coil. Symposium on Design, Test, Integration and Packaging of MEMS/MOEMS (DTIP), Cannes, 1-4 April 2014, 1-4. http://dx.doi.org/10.1109/dtip.2014.7056662

[23] Van Wageningen, D. and Staring, T. (2010) The Qi Wireless Power Standard. 14th International Power Electronics and Motion Control Conference (EPE/PEMC), Ohrid, 6-8 September 2010, S15-25-S15-32. http://dx.doi.org/10.1109/epepemc.2010.5606673

[24] Wireless Power Consortium, System Description, Wireless Power Transfer Volume I: Low Power, Part 1: Interface Definition (2013). http://www.wirelesspowerconsortium.com/downloads/wireless-power-specification-part-1.html

[25] Grover, F.W. (1946) Inductance Calculations. D. Van Nostrand Company, New York.

[26] Greenhouse, H.M. (1974) Design of Planar Rectangular Microelectronic Inductors. IEEE Transactions on Parts, Hybrids, and Packaging, 10, 101-109. http://dx.doi.org/10.1109/TPHP.1974.1134841

[27] MATLAB. http://fr.mathworks.com/products/matlab/

[28] Advanced Design System. http://www.keysight.com/en/pc-1297113/advanced-design-system-ads?cc=FR\&lc=fre

[29] Peters, C. and Manoli, Y. (2008) Inductance Calculation of Planar Multi-Layer and Multi-Wire Coils: An Analytical Approach. Sensors and Actuators A: Physical, 145-146, 394-404. http://dx.doi.org/10.1016/j.sna.2007.11.003

[30] Sun, X., Peng, X., Zheng, Y., Li, X. and Zhang, H. (2014) A 3-D Stacked High-Q PI-Based MEMS Inductor for Wireless Power Transmission System in Bio-Implanted Applications. Journal of Microelectromechanical Systems, 23, 888-898. http://dx.doi.org/10.1109/JMEMS.2013.2297627

[31] HD-4100 Series. http://www.hdmicrosystems.com/ec/liquid-polyimides-and-pbo-precursors/products/sub-products/hd-4100-series.html

[32] PI-2600 Series-Low Stress Applications. http://www.hdmicrosystems.com/ec/liquid-polyimides-and-pbo-precursors/products/sub-products/pi-2600-series.html

[33] BQ51050b. http://www.ti.com/lit/ds/symlink/bq51050b.pdf

[34] WR483250-15M2-XX. http://www.mouser.com/pdfdocs/tdkWR48325015M2XX120215.pdf 